\title{
LEARNING AND COOPERATIVE MULTIMODAL HUMANOID ROBOTS
}

\author{
Ruediger Dillmann \\ IRF, Karlsruhe University, Haid-und-Neu-Str. 7, Karlsruhe, Germany
}

In this talk a brief overview on the German Collaborative Research Center on Humanoid Robots is given. The research topics focused by the collaborative research center are multimodal man machine interaction, learning of motion, skills and tasks, man-robot cooperation, perception and action and mechatronics and controls for a human like torus consisting of 2 redundant arms, 2 dexterous 5-finger hands, a head with eyes and ears, a neck and a spine.

Over a period of 10 years it is envisaged to elaborate and establish basic methodologies and technology for humanoid robots to act and cooperate close with humans in a dayly living environment. An integrated perspective towards continuous humanoid interaction gaining multimodality adaptivity, redundancy and flexibility is proposed.

Programming, cooperation and interaction with a humanoid robot is assumed to be multimodal which means to let the user program the robot simply by speech, gesture or demonstrating a task. The robot observes, interprets and then tries to imitate and to learn the performed user action.

On the basis of a redundant humanoid 2 arms type, a robot system equipped with an active stereo head and a microphone array observation of the demonstrated task is realised as a first prototype using an active vision system. Grasping of objects is detected with the help of data gloves and active vision. The system interprets and stores the observed actions, segments them into meaningful sequences in a given context. Due to sensor errors and the complexity of the intended interaction, the system generates 
queries concerning intention, manipulation and objects. Motion catch techniques in combination with learning capabilities are combined towards imitation learning and active observational learning strategies.

The project will be outlined and actual research work and results will be discussed. 